Article

\title{
Temperature-Dependent X-ray Diffraction Measurements of Infrared Superlattices Grown by MBE
}

\author{
Charles J. Reyner*, Arnold M. Kiefer, Gamini Ariyawansa, Joshua M. Duran \\ and John E. Scheihing \\ Air Force Research Laboratory, Wright-Patterson Air Force Base, Dayton, OH 45433 USA; \\ arnold.kiefer@us.af.mil (A.M.K.); gamini.ariyawansa.2@us.af.mil (G.A.); joshua.duran.2@us.af.mil (J.M.D.); \\ john.scheihing@us.af.mil (J.E.S.) \\ * Correspondence: charles.reyner.1@us.af.mil; Tel.: +937-713-8358; Fax: +937-255-6942 \\ Academic Editor: Paul J. Simmonds \\ Received: 11 October 2016; Accepted: 15 November 2016; Published: 17 November 2016
}

\begin{abstract}
Strained-layer superlattices (SLSs) are an active research topic in the molecular beam epitaxy (MBE) and infrared focal plane array communities. These structures undergo a $>500 \mathrm{~K}$ temperature change between deposition and operation. As a result, the lattice constants of the substrate and superlattice are expected to change by approximately $0.3 \%$, and at approximately the same rate. However, we present the first temperature-dependent X-ray diffraction (XRD) measurements of SLS material on GaSb and show that the superlattice does not contract in the same manner as the substrate. In both InAs/ $\operatorname{InAs}_{0.65} \mathrm{Sb}_{0.35}$ and $\operatorname{In}_{0.8} \mathrm{Ga}_{0.2} \mathrm{As}_{\mathrm{InAs}} \mathrm{In}_{0.65} \mathrm{Sb}_{0.35} \mathrm{SLS}$ structures, the apparent out-of-plane strain states of the superlattices switch from tensile at deposition to compressive at operation. These changes have ramifications for material characterization, defect generation, carrier lifetime, and overall device performance of superlattices grown by MBE.
\end{abstract}

Keywords: Type-II superlattice; MWIR; infrared detectors; InAs/InAsSb; InGaAs/InAsSb; XRD; temperature dependence

\section{Introduction}

Strained layer superlattices (SLSs) are an area of interest for large area infrared focal plane arrays. In comparison to $\mathrm{HgCdTe}$, SLS structures can be deposited on inexpensive substrates, do not require extensive safety modifications for the deposition of mercury, and can take advantage of commercial foundries. As a result, multiple academic [1-3], industrial [4,5], and government groups [6-9] have researched these structures using molecular beam epitaxy (MBE). The original structures consisted of InAs/InGaSb [10], which were followed by gallium-free designs (InAs/InAsSb) [1]. Newer versions include InGaAs/InAsSb ternary SLS [11] and InAs/AlAs/AlSb/InAsSb W-structures [6,12]. The overall performance of SLS-based infrared photodetectors has improved to the point where they have similar dark current (Rule 07) [13] and external quantum efficiency to those based on HgCdTe. However, SLS still has not outperformed HgCdTe, most likely because of some combination of minority carrier lifetime [1], vertical minority carrier mobility [14], and low absorption coefficient [6,15]. Improvements to these characteristics will require further refinements in superlattice modeling, device design, and material deposition.

Modeling and characterization of SLS structures typically take place at $80-300 \mathrm{~K}$, and as a result, almost all strain calculations occur in this temperature region as well. However, materials expand and contract with temperature change and the linear coefficient of thermal expansion (CTE) for bulk III/V materials is $1-5 \times 10^{-5} \AA / \mathrm{K}$ [16]. While the linear CTE is small, the temperature change between material deposition ( $650-725 \mathrm{~K})$, ex situ characterization ( $298 \mathrm{~K})$, and operation ( $80-120 \mathrm{~K})$ is rather 
large. This temperature swing leads to changes in lattice constant on the order of $0.3 \%$, which is far greater than the lattice mismatch of the superlattice to the substrate at room temperature $(<0.1 \%)$.

In this letter, we present the first temperature-dependent $X$-ray diffraction (XRD) measurements of lattice constant for both InAs/ InAs ${ }_{0.65} \mathrm{Sb}_{0.35}$ and $\operatorname{In}_{0.8} \mathrm{Ga}_{0.2} \mathrm{As}_{\mathrm{In}} \mathrm{Ins}_{0.65} \mathrm{Sb}_{0.35} \mathrm{SLS}$ structures. We show that while the GaSb substrate behaves mostly according to theoretical models, the superlattice structures do not necessarily follow. The CTE for both structures is considerably smaller than that of bulk material, and in the case of the ternary superlattice, is almost zero. The ramifications on the strain state, its sensitivity to modeling conditions, and the difficulty of meeting the "zero stress" strain condition in MBE are discussed [17], as these are important considerations for the MBE user community.

\section{Results}

Temperature dependent XRD scans of InAs/InAs $0.65 \mathrm{Sb}_{0.35}$ (18 ML/6 ML) superlattices are shown in Figure 1. For clarity, the scans are split into the substrate/superlattice peak (upper frame), the $\mathrm{SL}-1$ peak (lower left frame), and the $\mathrm{SL}+1$ peak (lower right frame). Faint Pendellösung fringes are found on all three peaks and across all temperatures, indicating reasonable material quality. The GaSb substrate is identified as the highest, sharpest peak in the upper frame (annotated). Its location monotonically shifts to a higher angle with temperature as the substrate contracts with decreasing temperature. Meanwhile, the superlattice peak overlaps the substrate peak across the entire temperature range, and its location can be somewhat identified at the temperature extremes as a shoulder. At intermediate temperature (e.g., $248 \mathrm{~K}$ ), it is very difficult to absolutely identify the superlattice peak location. However, it is evident that the SL 0 peak switches from the right side of the substrate to the left as the sample cools; this is typically, albeit incorrectly, considered to be a change of the superlattice strain state from tensile to compressive [17]. The SL -1 and SL +1 peak locations also move in concert, indicating that the average out-of-plane lattice constant of the superlattice is changing but that the superlattice period is not necessarily changing at the same rate. The out-of-plane strains, based on a full elastic approach, are $-0.039 \%$ at $298 \mathrm{~K}$ and $-0.020 \%$ at $173 \mathrm{~K}$. The full width at half-maximum (FWHM) of the SL +1 peak is 81 arcsec at $298 \mathrm{~K}$ in $\omega-2 \theta$ notation.
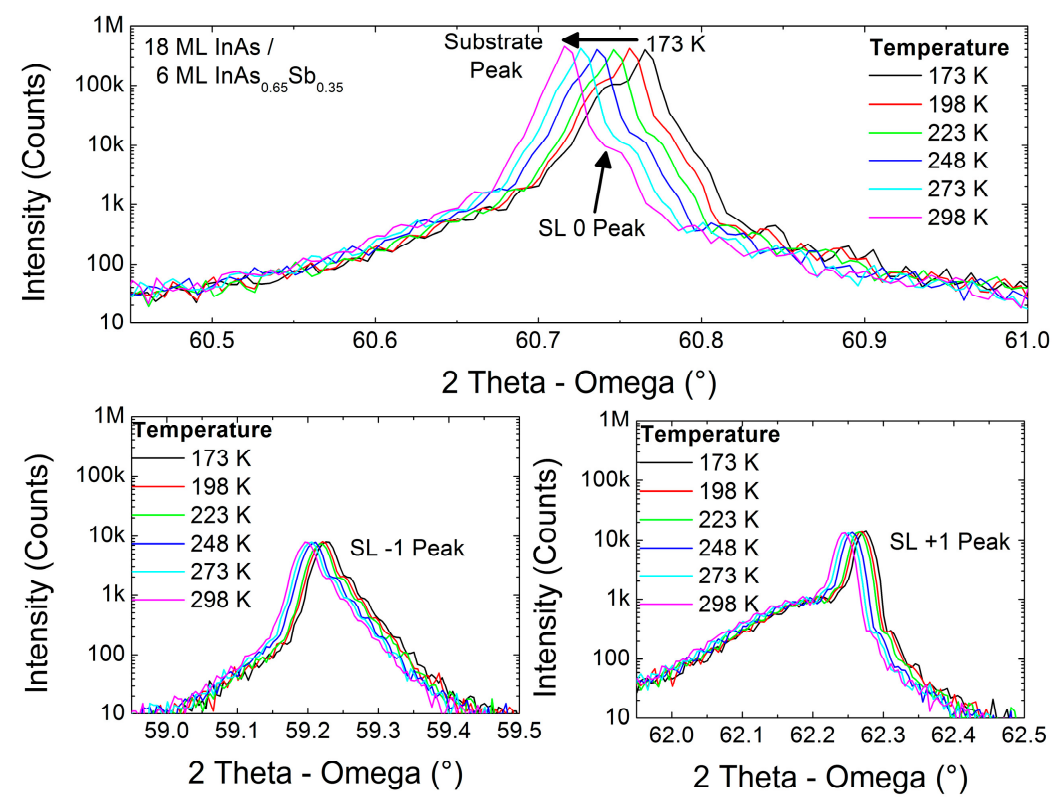

Figure 1. (Upper frame) X-ray diffraction (XRD) scans of the substrate and InAs/InAsSb SL 0 peak from $173-298 \mathrm{~K}$. The substrate and superlattice have approximately the same average out-of-plane lattice constant. (Lower left frame) The SL -1 peak of the superlattice. Pendellösung fringes are evident across temperature. (Lower right frame) The $\mathrm{SL}+1$ peak of the superlattice. The peak behaves similarly to the SL -1 peak. 
Temperature-dependent XRD scans of $\operatorname{In}_{0.8} \mathrm{Ga}_{0.2} \mathrm{As} / \mathrm{InAs}_{0.65} \mathrm{Sb}_{0.35}$ (8 ML/9 ML) superlattices are shown in Figure 2. Pendellösung fringes are not evident in this sample, although it is possible to see them at lower temperatures. Material quality could be the cause of the smeared fringes, although it is not clear if this was caused by strain-induced segregation of gallium or antimony [18], clustering caused by lower gallium adatom migration [19], or something else. However, the substrate and superlattice peaks are readily identifiable in the upper frame. The substrate peak location moves monotonically with temperature, much like the previous sample. The superlattice peak location barely changes, which indicates that the average out-of-plane lattice constant of the superlattice is not changing. The SL -1 and $\mathrm{SL}+1$ peaks exhibit similar behavior, neither moving in concert nor moving apart. The out-of-plane strains, based on a full elastic approach, are $-0.258 \%$ at $298 \mathrm{~K}$ and $-0.191 \%$ at $173 \mathrm{~K}$. The FWHM of the SL +1 peak is 74 arcsec at $298 \mathrm{~K}$ in $\omega-2 \theta$ notation.
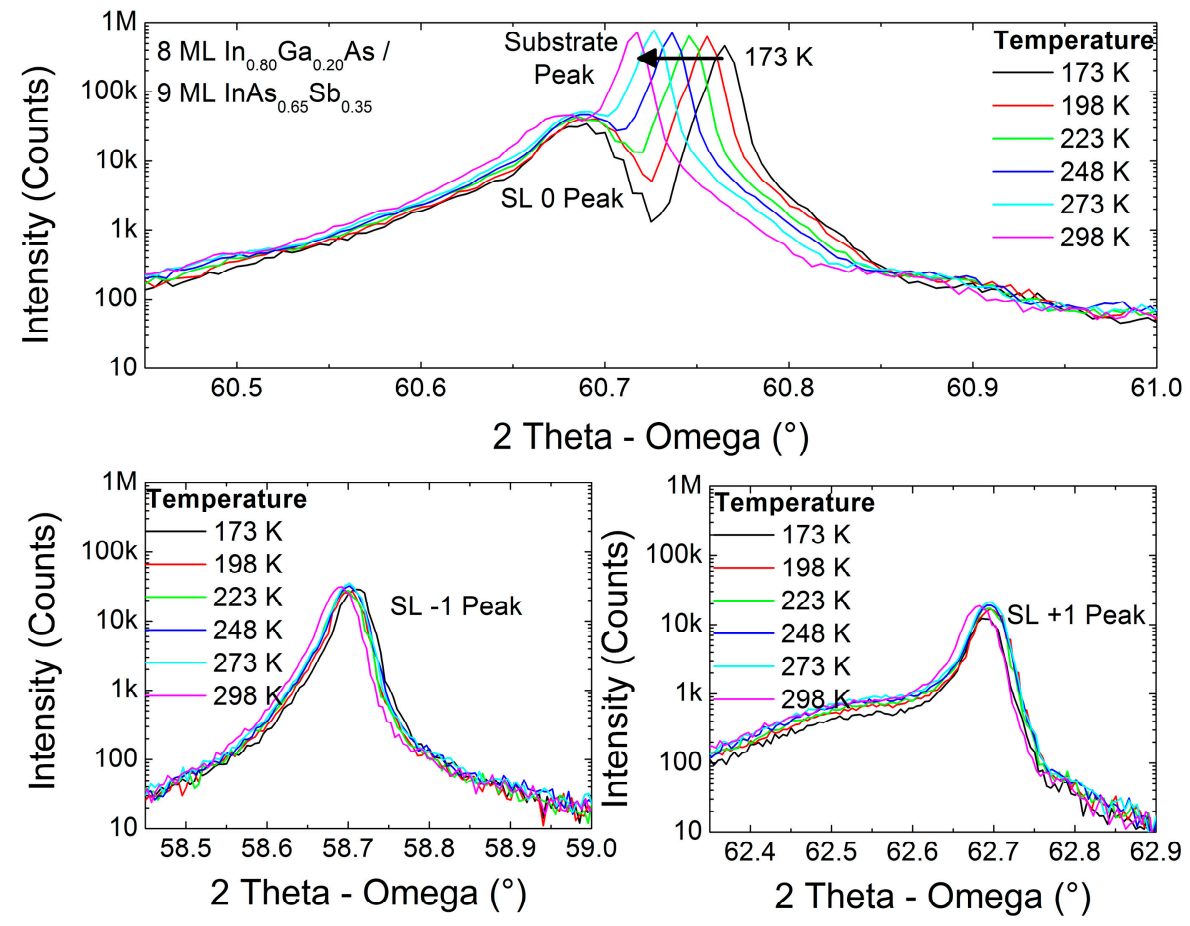

Figure 2. (Upper frame) XRD scans of the substrate and InGaAs/InAsSb SL 0 peak from 173-298 K. The substrate and superlattice remain compressively strained throughout. (Lower left frame) The SL -1 peak of the superlattice. The peak location does not change monotonically. (Lower right frame) The SL +1 peak of the superlattice. The peak behaves similarly to the SL -1 peak.

The lattice constant of $\mathrm{GaSb}$ was calculated using both experimental data and empirical/theoretical models [16,20-22]. The results are found in Figure 3. The experimental values for both samples are almost identical, and can be found within the bounds of the models. A linear fit is performed across the temperature range, which indicates that the linear CTE for GaSb is $3.46 \times 10^{-5} \AA / \mathrm{K}$, with an intercept of $6.09642 \AA$ at $298 \mathrm{~K}$. Vurgaftman uses a value of $4.72 \times 10^{-5} \AA / \mathrm{K}$, but the differences could be explained by substrate doping or improvements in substrate quality $[16,21]$. The Nilsen model used a fourth-order polynomial and was performed over a different temperature range (305-819 K), which could explain the larger error at low temperatures [20]. Nilsen's linear CTE estimate is $4.37 \times 10^{-5} \AA / \mathrm{K}$, which is slightly closer to our estimate. The Adachi model is the closest, with a linear CTE of $3.87 \times 10^{-5} \AA / K$, and an intercept of $6.09593 \AA$ at $298 \mathrm{~K}$ [22]. 


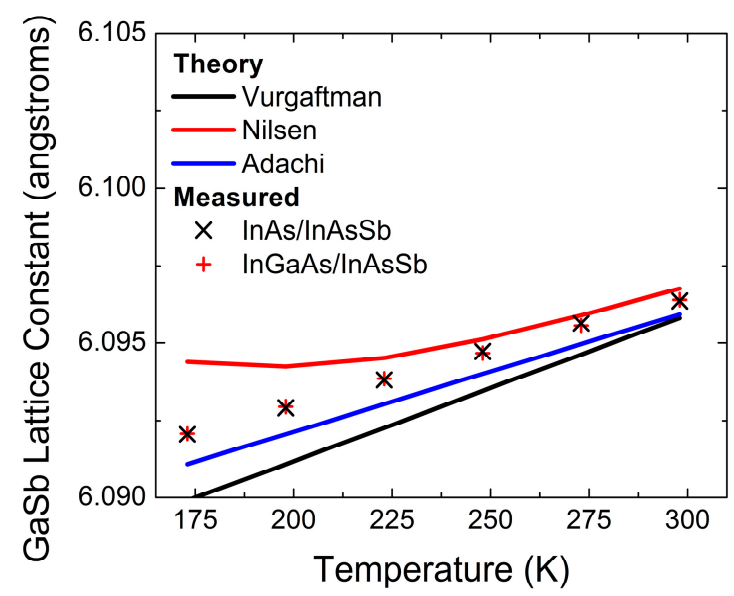

Figure 3. GaSb lattice constant based on models from the literature, as well as measured values from both samples (labeled by their superlattice).

The average out-of-plane lattice constants of the superlattices are plotted in Figure 4. While the $\operatorname{In}_{0.8} \mathrm{Ga}_{0.2} \mathrm{As} / \mathrm{InAs}_{0.65} \mathrm{Sb}_{0.35}$ sample had a clear superlattice peak that could be separated, the InAs/ $/ n \mathrm{As}_{0.65} \mathrm{Sb}_{0.35}$ sample did not. In this instance, the SL -1 and $\mathrm{SL}+1$ peaks were converted into reciprocal lattice units, and an average was taken to determine the SL 0 location. These measured values are compared to the theoretical values calculated from a full elastic approach, an average lattice approach, a strained average lattice approach, and NRL MultiBands [21]. The first three approaches can be found in Ekins-Daukes et al. as Equation 3, Equation 1, and Equation $4(v=0.35)$ [17] and the average lattice approach is the most commonly found in the MBE user community. The $\mathrm{In}_{0.8} \mathrm{Ga}_{0.2} \mathrm{As} / \mathrm{InAs}_{0.65} \mathrm{Sb}_{0.35}$ sample exhibits almost no change in average out-of-plane lattice constant (effective linear CTE near zero). Meanwhile, the InAs/InAs $\mathrm{As}_{0.65} \mathrm{Sb}_{0.35}$ sample exhibits some change in lattice constant, and the measured linear CTE is $2.14 \times 10^{-5} \AA / \mathrm{K}$ while the theoretical elastic value is $2.16 \times 10^{-5} \AA / \mathrm{K}$. The other models exhibit values between $2.8 \times 10^{-5}$ to $3.3 \times 10^{-5} \AA / \mathrm{K}$. The ternary superlattice also exhibits similar slopes for both the average lattice and strained average lattice calculations, unlike the gallium-free design. The lines are effectively parallel because of the similar amounts of strain and the elastic properties of each layer. The strain balancing of the ternary superlattice, as opposed to strain-thickness balancing with InAs, is unique amongst medium wavelength infrared (MWIR) superlattice designs, and provides more ability to tune the bandgap while maintaining the "zero stress" condition.
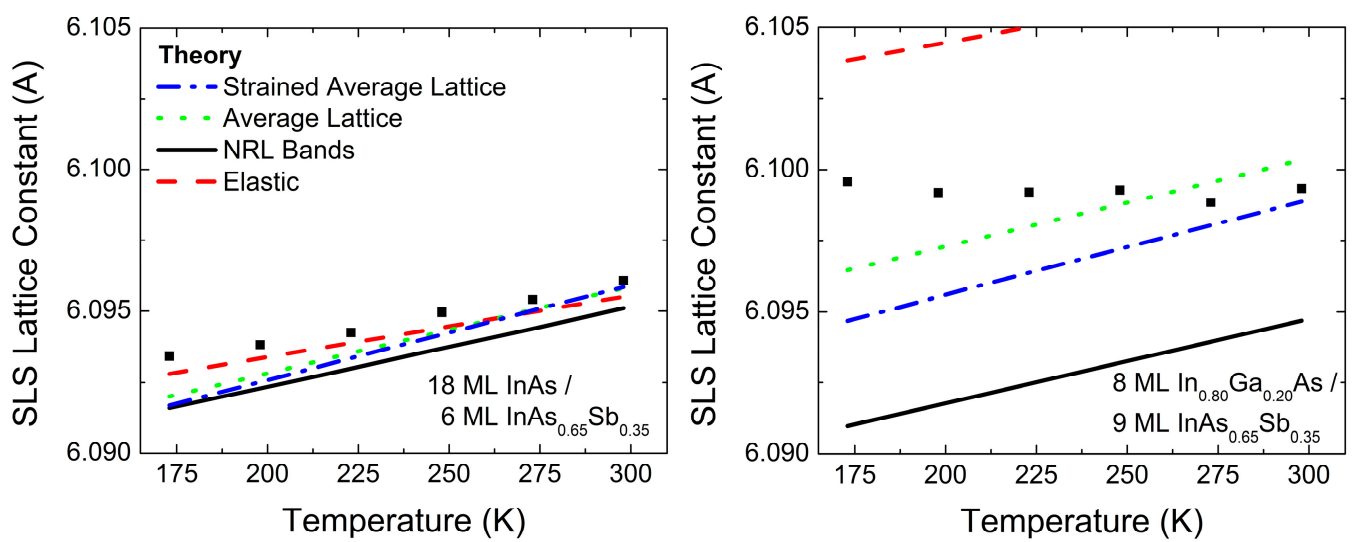

Figure 4. The Strained-layer superlattice (SLS) average out-of-plane lattice constant for InAs/InAsSb (left) and InGaAs/InAsSb (right). Measured values are shown as squares. Various theoretical values are given based on material models from Adachi (elastic and average lattice methods) as well as Vurgaftman. 
Finally, the superlattice period was calculated based on the separation between the SL -1 and $\mathrm{SL}+1$ peaks across temperature. Theoretical calculations by all four approaches predict a monotonic decrease in the period with decreasing temperature, but this was not observed in either measured sample, even when the period was verified against higher order peaks (e.g., SL 2 peaks). The most likely causes of this discrepancy is the small change in $2 \theta$ peak separation $\left(0.002^{\circ}\right)$ given the width of the SL peaks and the resolution of the diffractometer. Given the near constant period and relatively small changes in strain over temperature, the bandgap shift of SLSs can be mostly attributed to changes in the bandgap of the constituent layers (e.g. InAs, $\operatorname{InAs}{ }_{0.65} \mathrm{Sb}_{0.35}$, etc.).

\section{Discussion}

The results of this study demonstrate that the out-of-plane lattice constants of InAs/InAs $\mathrm{In}_{0.65} \mathrm{Sb}_{0.35}$ and $\operatorname{In}_{0.8} \mathrm{Ga}_{0.2} \mathrm{As} / \mathrm{InAs}_{0.65} \mathrm{Sb}_{0.35}$ superlattices change at different rates versus temperature than the $\mathrm{GaSb}$ substrate, and that it is extremely difficult to measure changes in period across temperature. In the case of these SLS structures, the difference in the lattice constant mismatch at the deposition temperature could conceivably be responsible for the lower quality material in $\operatorname{In}_{0.8} \mathrm{Ga}_{0.2} \mathrm{As}_{\mathrm{s}} / \mathrm{InAs}_{0.65} \mathrm{Sb}_{0.35}$. The experiment also shows why the average lattice constant method is so popular in the MBE user community, in that it has the best fit to experiment for both superlattices at room temperature. Since MBE users want to lattice-match the in-plane lattice constant with the substrate at the deposition temperature-the "zero stress" condition-this leads to inaccurate calculations and superlattice designs, which require a full elastic treatment. Further studies using in situ XRD [23], across various superlattice compositions, and at higher ex situ measurement temperatures-as well as asymmetric scans-should help to elucidate the exact linear CTE, elastic properties, and strain state at the deposition temperature. Unfortunately, even small changes in the linear CTE of these materials lead to very large changes in the "zero stress" condition. A commonly used 18/6 ML InAs/InAs ${ }_{0.6} \mathrm{Sb}_{0.4}$ superlattice [14] would require a $100 \mathrm{~K}$ change in deposition temperature to match the "zero stress" condition if the GaSb CTE parameters were changed from those in Adachi to the ones calculated here. These changes in conditions also assume perfect compositional and temperature uniformity across the substrate, which is not currently achievable [24]. It is also evident that metalorganic chemical vapor deposition (MOCVD) might require different SLS composition/period combinations to meet the "zero stress" condition, given the higher substrate temperatures necessary for precursor decomposition [25].

\section{Materials and Methods}

The SLS structures (500 nm thick) were deposited on lightly doped n-type GaSb (100) substrates using a Veeco Gen930 solid source molecular beam epitaxy reactor (Veeco, New York, NY, USA). These structures were designed to have the same bandgap [11], which would enable a fair comparison of absorption across each and ensure similar substrate temperatures during deposition. The deposition followed the standard III/V MBE deposition process, where the substrate was heated to $350{ }^{\circ} \mathrm{C}$ before applying an $\mathrm{Sb}_{2}$ overpressure. After surface oxide removal at $530{ }^{\circ} \mathrm{C}$, the samples were cooled to $510{ }^{\circ} \mathrm{C}$ and a GaSb buffer was grown. Details of the sample growth process may be found elsewhere [11], but care was taken to limit the V/III atomic flux ratio to as low of a number as possible. Early calibration samples were Group III-rich in some cases, which established the lower limit. After deposition, the samples were characterized using standard material characterization at room temperature, including atomic force microscopy, difference interference microscopy, Fourier-transform infrared spectroscopy, photoluminescence, and XRD. These results indicated that all samples were deposited as designed. The XRD measurements were taken on a PANalytical Empyrean X-ray diffractometer using a hybrid monochromator $\left(\mathrm{Cu} \mathrm{K \alpha} \alpha_{1}\right)$ on the incident beam and a triple-axis analyzer $\left(0.0033^{\circ}\right.$ acceptance angle) with proportional detector on the diffracted beam. The temperature stage was a DCS 350 (Anton Paar, Graz, Austria) domed cooling stage, which was configured for cryogenic operation from $173 \mathrm{~K}$ to $298 \mathrm{~K}$. The X-ray beam and detector were aligned when the stage was at room temperature, and then aligned on the sample's substrate peak by optimizing $\omega, 2 \theta-\omega$, and $\chi$ iteratively. 
A symmetric $2 \theta-\omega$ scan was then measured and the stage temperature lowered. After each temperature change, the beam was realigned on the substrate by changing $\omega$ and $2 \theta-\omega$ to account for changes in the stage's orientation/position as it cooled. It should be noted that the $2 \theta$ angle is recorded instead of the $\omega$ angle, which is more commonly reported, because sample tilts do not affect the $2 \theta$ angle directly. Once the full scan was complete, the stage was warmed back to $25^{\circ} \mathrm{C}$, and an additional short scan was performed to verify repeatability and that nothing else had changed in the system. The scans were repeatable both in peak location $(2 \theta-\omega)$ and peak intensity (counts per second). All scans were of the 004 reciprocal lattice point, and the X-ray wavelength is $1.540598 \AA$, per The manufacturer.

\section{Conclusions}

In conclusion, we provide the first temperature-dependent XRD measurements of SLS on GaSb grown by MBE. The extracted GaSb lattice constant matches the empirical models to within $0.1 \%$. Meanwhile, the superlattice structures exhibit little change in period or average out-of-plane lattice constant across a $125 \mathrm{~K}$ range, especially in relation to commonly used approximations in the MBE community. Furthermore, we show that the apparent strain state of the out-of-plane superlattice is highly dependent on measurement temperature and is not necessarily consistent with the strain state at the growth $(>650 \mathrm{~K})$ or operating $(80-120 \mathrm{~K})$ temperatures. Indeed, "zero stress" lattice-matching of superlattices during deposition is not trivial for high-quality MBE deposition.

Acknowledgments: This work was funded by the Air Force Research Laboratory, Sensors Directorate under project "III-V Focal Plane Array Development Using Novel Superlattices", as well as recapitalization funds for the temperature dependent stage. The authors would like to acknowledge the contributions from the scientists/engineers at the Naval Research Laboratory (NRL) by providing us with the NRL MULTIBANDS ${ }^{\text {TM }}$ software which was critical for the design/modeling of the superlattice structures. The authors would also like to acknowledge Baolai Liang and Diana L. Huffaker for support at UCLA/CNSI MBE facility.

Author Contributions: Charles J. Reyner, Arnold M. Kiefer, Gamini Ariyawansa, Joshua M. Duran, and John E. Scheihing conceived and designed the experiment; Gamini Ariyawansa, Joshua M. Duran, and John E. Scheihing modeled the superlattice design; Charles J. Reyner fabricated the samples; Gamini Ariyawansa, Joshua M. Duran, and John E. Scheihing verified the superlattice designs; Charles J. Reyner and Arnold M. Kiefer measured the samples using XRD; Charles J. Reyner and Arnold M. Kiefer analyzed the data; and Charles J. Reyner wrote the original draft.

Conflicts of Interest: The authors declare no conflicts of interest.

\section{References}

1. Steenbergen, E.H.; Connelly, B.C.; Metcalfe, G.D.; Shen, H.; Wraback, M.; Lubyshev, D.; Qiu, Y.; Fastenau, J.M.; Liu, A.W.; Elhamri, S.; et al. Significantly improved minority carrier lifetime observed in a long-wavelength infrared III-V type-II superlattice comprised of InAs/InAsSb. Appl. Phys. Lett. 2011, 99, 251110-251113. [CrossRef]

2. Hoang, A.M.; Chen, G.; Chevallier, R.; Haddadi, A.; Razeghi, M. High performance photodiodes based on InAs/InAsSb type-II superlattices for very long wavelength infrared detection. Appl. Phys. Lett. 2014, 104. [CrossRef]

3. Plis, E.A.; Schuler-Sandy, T.; Ramirez, D.A. Dark current reduction in InAs/InAsSb superlattice mid-wave infrared detectors through restoration etch. Electron. Lett. 2015, 51, 2009-2010. [CrossRef]

4. Klipstein, P.C. XBnn and XBpp infrared detectors. J. Cryst. Growth 2015, 425, 351-356. [CrossRef]

5. Rhiger, D.R.; Smith, E.P.; Kolasa, B.P.; Kim, J.K.; Klem, J.F.; Hawkins, S.D. Analysis of III-V Superlattice nBn Device Characteristics. J. Electron. Mater. 2016, 45, 4646-4653. [CrossRef]

6. Olson, B.V.; Kim, J.K.; Kadlec, E.A.; Klem, J.F.; Hawkins, S.D.; Coon, W.T.; Fortune, T.R.; Tauke-Pedretti, A.; Cavaliere, M.A.; Shaner, E.A. Optical and electrical properties of narrow-bandgap infrared W-structure superlattices incorporating AlAs/AlSb/AlAs barrier layers. Appl. Phys. Lett. 2016, 108, 252104. [CrossRef]

7. Donetsky, D.; Svensson, S.P.; Vorobjev, L.E.; Belenky, G. Carrier lifetime measurements in short-period InAs/GaSb strained-layer superlattice structures. Appl. Phys. Lett. 2009, 95, 212104. [CrossRef] 
8. Ting, D.Z.-Y.; Hill, C.J.; Soibel, A.; Keo, S.A.; Mumolo, J.M.; Nguyen, J.; Gunapala, S.D. A high-performance long wavelength superlattice complementary barrier infrared detector. Appl. Phys. Lett. 2009, 95, 023508. [CrossRef]

9. Connelly, B.C.; Metcalfe, G.D.; Shen, H.; Wraback, M.; Canedy, C.L.; Vurgaftman, I.; Melinger, J.S.; Affouda, C.A.; Jackson, E.M.; Nolde, J.A.; et al. Investigation of Trap States in Mid-Wavelength Infrared Type II Superlattices Using Time-Resolved Photoluminescence. J. Electron. Mater. 2013, 42, 3203-3210. [CrossRef]

10. Fuchs, F.; Weimer, U.; Pletschen, W.; Schmitz, J.; Ahlswede, E.; Walther, M.; Wagner, J.; Koidl, P. High performance InAs $/ \mathrm{Ga}_{1-\mathrm{x}} \mathrm{In}_{\mathrm{x}} \mathrm{Sb}$ superlattice infrared photodiodes. Appl. Phys. Lett. 1997, 71, 3251-3253. [CrossRef]

11. Ariyawansa, G.; Reyner, C.J.; Steenbergen, E.H.; Duran, J.M.; Reding, J.D.; Scheihing, J.E.; Bourassa, H.R.; Liang, B.L.; Huffaker, D.L. InGaAs/InAsSb strained layer superlattices for mid-wave infrared detectors. Appl. Phys. Lett. 2016, 108, 022106. [CrossRef]

12. Canedy, C.L.; Aifer, E.H.; Vurgaftman, I.; Tischler, J.G.; Meyer, J.R.; Warner, J.H.; Jackson, E.M. Antimonide Type-II “W” Photodiodes with Long-Wave Infrared R 0 A Comparable to HgCdTe. J. Electron. Mater. 2007, 36, 852-856. [CrossRef]

13. Tennant, W.E. "Rule 07" revisited: Still a good heuristic predictor of $\mathrm{p} / \mathrm{n} \mathrm{HgCdTe}$ photodiode performance? J. Electron. Mater. 2010, 39, 1030-1035. [CrossRef]

14. Ting, D.Z.; Soibel, A.; Gunapala, S.D. Hole effective masses and subband splitting in type-II superlattice infrared detectors. Appl. Phys. Lett. 2016, 108, 183504. [CrossRef]

15. Ariyawansa, G.; Reyner, C.J.; Duran, J.M.; Reding, J.D.; Scheihing, J.E.; Steenbergen, E.H. Unipolar infrared detectors based on InGaAs/InAsSb ternary superlattices. Appl. Phys. Lett. 2016, 109, 021112. [CrossRef]

16. Vurgaftman, I.; Meyer, J.R.; Ram-Mohan, L.R. Band parameters for III-V compound semiconductors and their alloys. J. Appl. Phys. 2001, 89, 5815-5875. [CrossRef]

17. Ekins-Daukes, N.J.; Kawaguchi, K.; Zhang, J. Strain-Balanced Criteria for Multiple Quantum Well Structures and Its Signature in X-ray Rocking Curves. Cryst. Growth Des. 2002, 2, 287-292. [CrossRef]

18. Timm, R.; Lenz, A.; Eisele, H.; Ivanova, L.; Dähne, M.; Balakrishnan, G.; Huffaker, D.L.; Farrer, I.; Ritchie, D.A. Quantum ring formation and antimony segregation in GaSb/GaAs nanostructures. J. Vacuum Sci. Technol. B Microelectron. Nanometer Struct. 2008, 26, 1492-1503. [CrossRef]

19. Shapiro, J.N.; Lin, A.; Huffaker, D.L.; Ratsch, C. Potential energy surface of In and Ga adatoms above the (111)A and (110) surfaces of a GaAs nanopillar. Phys. Rev. B 2011, 84, 085322. [CrossRef]

20. Nilsen, T.A.; Breivik, M.; Myrvågnes, G.; Fimland, B.-O. Thermal expansion of GaSb measured by temperature dependent X-ray diffraction. J. Vacuum Sci. Technol. B 2010, 28, C3I17-C3I20. [CrossRef]

21. Lumb, M.P.; Vurgaftman, I.; Affouda, C.A.; Meyer, J.R.; Aifer, E.H.; Walters, R.J. Quantum wells and superlattices for III-V photovoltaics and photodetectors. Proc. SPIE 2012, 8471, 84710A.

22. Adachi, S. Properties of Group-IV, III-V and II-VI Semiconductors; John Wiley \& Sons Ltd.: Chichester, UK, 2005.

23. Proessdorf, A.; Hanke, M.; Jenichen, B.; Braun, W.; Riechert, H. Volmer-Weber growth of AlSb on Si(111). Appl. Phys. Lett. 2013, 102, 041601. [CrossRef]

24. Jackson, A.W.; Gossard, A.C. Thermal imaging of wafer temperature in MBE using a digital camera. J. Cryst. Growth 2007, 301-302, 105-108. [CrossRef]

25. Kuech, T.F.; Khandekar, A.A.; Rathi, M.; Mawst, L.J.; Huang, J.Y.T.; Song, X.; Babcock, S.E.; Meyer, J.R.; Vurgaftman, I. MOVPE growth of antimonide-containing alloy materials for long wavelength applications. J. Cryst. Growth 2008, 310, 4826-4830. [CrossRef]

(C) 2016 by the authors; licensee MDPI, Basel, Switzerland. This article is an open access article distributed under the terms and conditions of the Creative Commons Attribution (CC-BY) license (http://creativecommons.org/licenses/by/4.0/). 